\title{
TRATAMIENTO JURÍDICO DE LAS ENERGIAS RENOVABLES EN COLOMBIA: AHORRO ENERGÉTICO, EFICIENCIA ENERGÉTICA Y USO RACIONAL DE LA ENERGÍA ${ }^{1}$
}

Legal Treatment of Renewable Energies in Colombia: Energy Saving, Energy Efficiency and Rational Use of Energy

$\underline{\text { Milton José Pereira Blanco }}^{2}$

Fecha de recepción: 4 de noviembre de 2016

Fecha de aceptación: 13 de diciembre de 2016

SUMARIO: 1. Introducción; 2. Energías renovables: fundamento normativo; 3. Crisis energética y posibles salidas; 4 . Ahorro energético, uso racional y eficiente de la energía: concepto y régimen jurídico; 5. Los biocombustibles como estrategia de promoción del uso racional y eficiente de la energía; 6. Conclusiones; 7. Referencias bibliográficas.

1 El Presente trabajo constituye un avance del marco teórico de la investigación concluida, titulada Relación entre Energía, Medio Ambiente y Desarrollo Económico a partir del Análisis Jurídico de las Energías Renovables en Colombia del cual el autor es investigador principal, y fue dirigida por el Doctor en Derecho Carlos Javier Velásquez Muñoz. Este trabajo se encuentra adscrito a la línea de investigación denominada Asuntos públicos y administración de justicia del Grupo de Investigación en Derecho y Ciencia Política de la Universidad del Norte.

2 Profesor de Derecho Administrativo General, Teoría General del Derecho y Filosofía del Derecho I de la Universidad de Cartagena y de la Fundación Universitaria Colombo Internacional. Abogado y Licenciado en Filosofía (C). Magíster en Derecho, Universidad del Norte. Especialista en Derecho Contencioso Administrativo de la Universidad Externado de Colombia, y estudiante de la especialización en Derecho del Medio Ambiente de la misma Universidad. Correo electrónico: mpereira@unicolombo.edu.co y miltonjosepereirablanco@gmail.com. 


\section{COMO CITAR ESTE ARTÍCULO (APA 6)}

Pereira Blanco, Milton José (2017). Tratamiento jurídico de las energías renovables en Colombia: ahorro energético, eficiencia energética y uso racional de la energía. Revista Jurídica Mario Alario D’Filippo, IX (17), pág. 43-68.

\section{RESUMEN}

En este trabajo se sostiene como tesis central, que el Estado Colombiano, sí bien ha desarrollado tímidamente el tema de los biocombustibles como energías renovables, la propuesta frente a otras fuentes no convencionales ha sido deficiente, porque no ha estructurado herramientas eficaces para entregar competitividad dentro del mercado energético a las energías renovables, siendo pasivo el Estado pasivo frente a la protección ambiental en cuanto a este punto se refiere. Lo anterior a partir del siguiente problema: ¿Cual ha sido el tratamiento jurídico de las energías renovables en Colombia y cual ha sido el nivel de incidencia de estas, en la protección ambiental? La tesis expuesta implica sostener que la eficiencia energética se convierte en el elemento principal de las energías renovables, dado que a partir de este tipo de energía se protege el medio ambiente. De igual forma, se debe señalar que el sistema jurídico energético colombiano no incluye en la canasta energética a las energías renovables como componente central, por cuanto la normatividad nacional solo se limita a fomentar las energías renovables sin implementar reformas estructurales al sistema en aras de hacer de las energías renovables el elemento central de la economía colombiana.

\section{PALABRAS CLAVES}

Eficiencia energética, uso racional de la energía, ahorro energético, crisis energética

\section{ABSTRACT}

This paper argues that the Colombian State has timidly developed the topic of biofuels as renewable energies. The proposal against other non-conventional sources has been deficient because it has not structured effective tools to deliver competitiveness within Of the energy market to renewable energies, the passive State being passive in relation to environmental protection in this regard. The above from the following problem: What has been the legal treatment of renewable energies in Colombia and what has been the incidence of these in environmental protection? The thesis presented implies that energy efficiency becomes the main element of renewable energies, given that this type of energy protects the environment. Likewise, it should be noted that the Colombian energy legal system does not include renewable energies in the energy basket as a central component, since national regulations are limited to promoting renewable energies without implementing structural reforms to the system for the sake of renewable energies the central element of the Colombian economy.

\section{KEYWORDS}

Energy efficiency, rational use of energy, energy saving, energy crisis 


\section{INTRODUCCIÓN}

En este trabajo se analiza el tratamiento jurídico de las energías renovables en Colombia a partir del estudio del ahorro energético, la eficiencia energético y el uso racional de la energía. Lo anterior a partir del problema jurídico planteado, el cual hace referencia a $¿ C u a l$ ha sido el tratamiento jurídico de las energías renovables en Colombia y cual ha sido el nivel de incidencia de estas, en la protección ambiental?. Es importante empezar por decir que el sector energético desempeña un papel trascendental en la economía, por cuanto la energía es el fundamento del crecimiento y del desarrollo económico. Lo anterior no se puede mirar de manera aislada, ya que el crecimiento hay que entenderlo de manera sostenible, lo cual implica trabajar de la mano con el medio ambiente a través de la protección del mismo.

El sistema jurídico energético colombiano no incluye en la canasta energética a las energías renovables como componente central, por cuanto la normatividad nacional solo se limita a fomentar las energías renovables sin implementar reformas estructurales al sistema en aras de hacer de las energías renovables el elemento central de la economía colombiana.

La importancia de la incorporación de las energías renovables dentro de la canasta energética en competencia frente a las fuentes tradicionales radica en la mayor eficiencia energética de las primeras frente a las segundas. Así, la eficiencia energética en Colombia ha sido trabajada a partir de los siguientes enfoques normativos, así: 1. En relación directa con el PROURE. 2. Para las zonas interconectadas. 3. Fuentes no convencionales de energía con relación al cambio climático. 4. En relación con los biocombustibles. 5 . Para los alcoholes carburantes. 6. Para el Gas. 7 Para la Cogeneración.

La metodología, la misma aplicada para análisis de las situaciones, el estudio de los datos de situaciones, teniendo en cuenta la posibilidad de inferir descriptivamente ideas, al comparar información en los ámbitos de estudio.

Para dar respuesta al problema jurídico planteado, su contenido se ha dividido en cuatro apartes, ordenados de la siguiente manera: El primer aparte denominado: Crisis energética y posibles salidas. El segundo aparte se titula: Energías Renovables: Fundamento Normativo. El tercer aparte se abordó el tema: Ahorro Energético, Uso racional y Eficiente de la Energía. El cuarto y último aparte se trabajó el tema: Los Biocombustibles como estrategia de promoción del Uso racional y Eficiente de la Energía. Para llegar a las conclusiones planteadas fue necesario acudir a bibliografía nacional como internacional, incluido documentos oficiales. 


\section{ENERGÍAS RENOVABLES: FUNDAMENTO NORMATIVO}

En este a parte del presente trabajo, se analiza el alcance del concepto de las energías renovables y su fundamento jurídico en Colombia. Antes de definir la importancia de las fuentes de energías renovables, es oportuno señalar que se entiende por tales, y la doctrina en general entiende por ellas como aquellas fuentes que por su cantidad en relación a los consumos que los seres humanos pueden hacer de ellas son inagotables y su propio consumo no afecta el medio ambiente (Estrada Gasca \& Arancibia Bulnes, 2010, p 13).

Estas fuentes de energía son muy importantes, por cuanto son muy abundantes en el planeta, amén de no afectar el medio ambiente ${ }^{3}$, pues es tal la abundancia de las FER ${ }^{4}$ que baste decir que la energía solar recibida cada 10 días sobre la Tierra equivale a todas las reservas conocidas de petróleo, carbón y gas (Estrada Gasca \& Arancibia Bulnes, 2010, p 11).

En cuanto al contexto nacional las energías convencionales han sido de origen fósil (hidrocarburos líquidos y gaseosos), que se han empleado para la locomoción de vehículos terrestres, aeronaves, embarcaciones marinas, la industria, el uso doméstico o incluso la generación de energía eléctrica, en este último caso, concurrentemente las fuentes hidráulicas.

De acuerdo con Hernández Mendible, el principal beneficio que producen las energías renovables frente a las energías tradicionales, es que disminuyen la dependencia de estas, las van sustituyendo de forma progresiva y contribuyen a mantener la calidad de vida de las personas actuales, garantizando un ambiente ecológicamente más equilibrado, lo que permite preservar el ambiente sano de las generaciones futuras y contribuir a la disminución del calentamiento global.

Es importante señalar que las energías renovables se erigen, pues, como fuentes de carácter estratégico, tanto es así que en el preámbulo del anteproyecto de la Ley para el fomento de las energías renovables en España, se dejó claro que para una mayor seguridad energética y del suministro, son las únicas que cumplen con la necesidad de mitigar la contaminación y el cambio climático.

Para el caso colombiano, es preciso señalar como fundamento normativo frente al fomento del uso racional de la energía y las energías renovables la ley 697 de 2001 y la ley 1715 de 2014. La norma en mención no hace expresa alusión literal a las energías

3. Estrada Gasca y Arancibia Bulnes expresan que la producción de gases de efecto de invernadero, principalmente el dioxido de carbono debido al uso intensivo de los hidrocarburos, son los precursores del incremento de la temperatura media global y consecuentemente del llamado cambio climatico, con todas las consecuencias para los seres humanos que ello implica.

4. Fuentes de Energías Renovables. 
renovables, sin embargo le entrega un tratamiento de energías alternativas, por cuanto las trabaja como fuentes no convencionales de energía. la Ley 697 de 2001, señala:

(...) Fuentes No Convencionales: Para efectos de la presente ley son fuentes no convencionales de energía, aquellas fuentes de energía disponibles a nivel mundial que son ambientalmente sostenibles, pero que en el país no son empleadas o son utilizadas de manera marginal y no se comercializan ampliamente.

Lo anterior es claro, y para el caso Colombiano, según la ley 697 de 2001, la energía hidroeléctrica producida por las centrales hidroeléctricas no son energías renovables, ya que no es una energía ambientalmente sostenible, contrario a lo planteado no tan claramente por la UPME y el Ministerio de Minas y Energías.

La Ley 1715 de 2014 frente al tema de las fuentes no convencionales señala que:

(...) 16. Fuentes No Convencionales de Energía (FNCE). Son aquellos recursos de energía disponibles a nivel mundial que son ambientalmente sostenibles, pero que en el país no son empleados o son utilizados de manera marginal y no se comercializan ampliamente. Se consideran FNCE la energía nuclear o atómica y las FNCER. Otras fuentes podrán ser consideradas como FNCE según lo determine la UPME.

17. Fuentes No Convencionales de Energía Renovable (FNCER). Son aquellos recursos de energía renovable disponibles a nivel mundial que son ambientalmente sostenibles, pero que en el país no son empleados o son utilizados de manera marginal y no se comercializan ampliamente. Se consideran FNCER la biomasa, los pequeños aprovechamientos hidroeléctricos, la eólica, la geotérmica, la solar y los mares. Otras fuentes podrán ser consideradas como FNCER según lo determine la UPME.

De acuerdo con Tejeiro Gutiérrez, la Ley 697 de 2001, se constituye en el instrumento normativo más relevante sobre las FNCE en Colombia, toda vez que además de establecer un marco normativo de carácter especial sobre la materia, aporta las definiciones conceptuales sobre las FNCE aplicables al sector energético y declara de interés público al Uso Racional de la Energía (URE). (2011, p 142).

La ley 697 de 2001 y 1715 de 2014 materializan uno de los presupuestos centrales de la Constitución de 1991 en materia de derechos colectivos, representado en el derecho al ambiente sano, el cual lo encontramos en el artículo 79 y expresamente consagra:

Todas las personas tienen derecho a gozar de un ambiente sano. La ley garantizará la participación de la comunidad en las decisiones que puedan afectarlo.

Es deber del Estado proteger la diversidad e integridad del ambiente, conservar las áreas de especial importancia ecológica y fomentar la educación para el logro de estos fines. 
En síntesis, la utilización de las energías renovables se evita mayor contaminación al planeta, aunque es importante decir, solo a título de salvedad, que las energías alternativas también resultan afectivas para con el medio ambiente aunque en mucha menor proporción. Sobre el punto de las energías alternativas es oportuno traer a colación lo planteado por Ramírez Laureano, el cual nos dice que en el caso de la energía eólica, el viento es uno de los recursos renovables más atractivos, a pesar de su naturaleza intermitente y variable (Ramirez Laureano, E., \& otro, 2008, p 15).

Las fuentes renovables de energía revisten interés para el abastecimiento energético por razones medioambientales y geopolíticas. Es necesaria una tecnología que permita abaratar la producción para que sea económicamente rentable y accesible. En teoría, la energía renovable puede proporcionar un abastecimiento seguro, no contaminante y asequible, recurriendo a fuentes autóctonas sin riesgo de ruptura o agotamiento de las reservas.

No obstante, si se quiere alcanzar esta meta, será necesario tomar medidas especiales para ello. Uno de los principales obstáculos, además de las dificultades técnicas, es el elevado coste de las tecnologías sobre energías renovables en comparación con las tecnologías sobre combustibles fósiles. De ahí la necesidad de aplicar incentivos financieros adecuados para promocionarlas. En los sectores donde la tecnología está más avanzada, por ejemplo, la energía eólica, los costes cayeron de forma espectacular en el decenio anterior y lo siguen haciendo ahora.

Con unas inversiones adecuadas en investigación, desarrollo y demostración de tecnologías que permitan la comercialización de energías renovables a corto, medio y largo plazo, esas fuentes podrán contribuir a resolver de una forma aceptable desde el punto de vista medioambiental y económico muchos de los problemas a que se enfrenta el abastecimiento energético de Europa a largo plazo. El desarrollo total de las fuentes renovables de energía, por ejemplo, puede desempeñar un papel muy importante en la reducción de las emisiones de gases de efecto invernadero en la producción de electricidad. No obstante, para ello, será preciso introducir rápidamente medidas especiales, incentivos económicos y una campaña enérgica de marketing (Ciemat).

Las energías renovables son sumamente importantes frente al tema de la protección ambiental y la seguridad del suministro; De conformidad con lo anterior, los Estados son quienes tienen la obligación de prever, matizar la crisis y tomar con mucha anticipación las medidas correspondientes. A este efecto, las políticas que garanticen el efectivo mantenimiento e incremento de la investigación científica y tecnológica que desarrolle nuevas fuentes energéticas serán decisivas.

Para el caso Colombiano, la ley 697 de 2001, muy tímidamente, incorporó la promoción de la investigación para el desarrollo de fuentes alternativas no convencionales. Sin 
embargo la nueva ley 1715 de 2014 fui un poco mas allá frente al tema de la integración de las energías renovables al sistema eléctrico colombiano, sin embargo, sigue siendo un asunto tratado de manera muy tímida a partir de la promoción y actividades de fomento.

En este sentido, la ley 1715 de 2013 planteo como objeto el de promover el desarrollo y la utilización de las fuentes no convencionales de energía, principalmente aquellas de carácter renovable, en el sistema energético nacional, mediante su integración al mercado eléctrico, su participación en las zonas no interconectadas y en otros usos energéticos como medio necesario para el desarrollo económico sostenible, la reducción de emisiones de gases de efecto invernadero y la seguridad del abastecimiento energético.

De lo anterior se evidencia una relación indisoluble y necesaria entre la energía, medio ambiente y desarrollo economía. Es claro que la energía se requiere indefectiblemente para el desarrollo, sin embargo genera afectaciones al ambiente. La conexidad necesaria entre el medio ambiente ante la crisis energética (escasez o inseguridad en el suministro, insostenibilidad del sistema y afectaciones ambientales) surge como estrategia para no frenar el desarrollo a partir de la energías renovables.

\section{CRISIS ENERGÉTICA Y POSIBLES SALIDAS}

En este punto se analiza el porqué de la importancia de la energía y en qué estado nos encontramos frente al tema energético. Pues, de manera generalizada se tiene la certeza de la existencia de la crisis en el sector energético.

Los efectos de la crisis energética pueden ser vistos de dos puntos de vista: el histórico propiamente dicho y el histórico material. Desde el punto de vista histórico propiamente dicho, se hace referencia a la relación energía, medio ambiente y desarrollo a partir del concepto de escasez del suministro y dependencia de los combustibles fósiles, a partir del crecimiento ilimitado. El segundo punto de vista; es decir, el histórico material, estudia el recurso energético de mayor impacto ambiental.

En efecto, los dos parámetros que se han considerado en el pasado como fundamentales para detonar el consumo de energía son: el crecimiento demográfico y el crecimiento económico (Martínez Fernández, 2010, p 4). De igual forma, otros autores plantean que la disponibilidad de energía en cantidades suficientes, de calidad y a precios competitivos, constituye una condición indispensable para el desarrollo económico y social de los pueblos. Esta exigencia es aún mayor en la disponibilidad de electricidad, para la que se requiere una garantía de suministro del $100 \%$.

La crisis energética nos demuestra la falta de coherencia entre el tema energético y el tema ambiental, por tanto, para tratar de superar la crisis energética se debe trabajar por 
una regulación energética ambiental, ya que la crisis energética no solo implica escasez de las fuentes de energía primaria, sino también el deterioro ambiental.

El sector energético desempeña un papel trascendental en la economía, por cuanto la energía es el fundamento del crecimiento y del desarrollo económico. Lo anterior no se puede mirar de manera aislada, ya que el crecimiento hay que entenderlo de manera sostenible, lo cual implica trabajar de la mano con el medio ambiente a través de la protección del mismo.

El desarrollo implica, entonces, utilización de la energía, y el uso de la energía para que sea sostenible debe ser racional y eficiente. Así mismo, el desarrollo sostenible implica la utilización de energía limpia. Una de las tensiones más grave que nos espera frente a la crisis energética, entendiendo como tal el agotamiento de los combustibles fósiles, es que los países y las empresas que controlan los recursos fósiles tienen todo el interés del mundo en mantener la utilización de estos recursos, tal como lo demuestra la historia reciente del sector petrolero. Cuando se concreten estas tensiones mundiales, cada país, cada rincón del mundo tendrá que elaborar sus planes para afrontar esta situación de emergencia (Coderch, 2008, p 50).

En cuanto a la crisis energética, la "demanda mundial de energía primaria señala que en la actualidad un $87 \%$ de la energía que se consume es agotable, circunstancia que genera 1. Inseguridad respecto al abastecimiento; 2. Volatilidad de los precios (entre otros factores, por los mayores requerimientos de las economías emergentes como China e India, cuyos crecimientos superan ampliamente el promedio mundial), y 3. Degradación ambiental, principalmente la generación del cambio climático ${ }^{5}$.

La doctrina ha planteado unos parámetros que debemos considerarlos importantes a efectos de manejar el abastecimiento energético. El abastecimiento energético debe analizarse desde la triple perspectiva del coste, la seguridad y el impacto medioambiental. Por último, la preocupación por el impacto de la energía en el cambio climático obliga a moderar las emisiones de efecto invernadero.

Teniendo en cuenta lo dicho hasta aquí, el desarrollo del sector energético debe conciliar de manera razonable y prudente estos tres condicionantes. Desde el punto de vista de la seguridad del abastecimiento, influye la sustitución de unas energías por otra. Cuando las energías fósiles se usan preferentemente como combustible, tal como sucede con el carbón y el gas natural, pueden sustituirse unas por otras dentro de ciertos límites.

Frente a lo expuesto hasta aquí, es claro el papel fundamental de las energías renovables, por cuanto estas son el factor fundamental para sostener el enlace entre medio

5 Velasquez Muñoz, Carlos, Op cit, 2009, pp 70 
ambiente, desarrollo económico y energía. La importancia de las energías renovables radica en lo siguiente: 1 . Se extraen de fuentes que se regeneran de manera natural, 2. Son inagotables, 3. Energías limpias, y 4. No emiten GEI.

De acuerdo con Hernández Mendible, el principal beneficio que producen las energías renovables frente a las energías tradicionales, es que disminuyen la dependencia de estas, las van sustituyendo de forma progresiva y contribuyen a mantener la calidad de vida de las personas actuales, garantizando un ambiente ecológicamente más equilibrado, lo que permite preservar el ambiente sano de las generaciones futuras y contribuir a la disminución del calentamiento global (Hernández Mendible, 2013, 152).

En cuanto el caso colombiano, como muchos otros países, existe una gran dependencia del petróleo (37,5\%). Sin embargo, en este caso, las energías renovables representan una porción bastante grande, superior al $27 \%$ del suministro total de energía. Las energías renovables no solo son medios para combatir el cambio climático, son el componente central del mercado energético por cuanto son el tema central de la seguridad del suministro y la competitividad a partir de la eficiencia energética y la diversificación de las fuentes de energía.

El Estado Colombiano no tiene aún una propuesta estructural para efectos de la expansión de las energías renovables, más allá del fomento de las energías renovables como preocupación ambiental; sin embargo, tal como lo demostraremos más adelante, no se evidencia un esquema de trabajo jurídico, ni técnico para propender por la seguridad del suministro energético y diversificación de las fuentes a partir de las energías renovables, frente a la crisis energética.

La doctrina ha planteado a grandes rasgos las posibles salidas al problema energético ambiental, los cuales se resumen así: 1 . Ahorrar energía, 2. Diversificar las fuentes y 3. Desarrollar nuevos procesos (Iranzo Martinez, J. E y Colinas Gonzalez M, 2008, p 456).

Es importante señalar entre ahorro energetico, eficiencia energetica y uso racional de la energía, entiendo el ahorro energético, como la energía no consumida, por cuanto no se hace necesario su consumo. La eficiencia energética hace referencia al menor impacto ambiental, y el uso racional de la energía, a su mayor aprovechamiento.

En cuanto a los conceptos de ahorro de energía, eficiencia energética y uso racional de la energía existe una diferencia; sin embargo, todo va concatenado hacia la protección del medio ambiente.

6 Iranzo Martinez, Juan Emilio y Colinas Gonzalez Manuel, Op cit, 45 
Para el caso colombiano, el Ministerio de Minas y Energías, de conformidad con lo establecido en la ley 697 de 2001 y en la Ley 99 de 1993, expidió la resolución No. 180919 de junio de 2010, por la cual se adopta el plan de acción indicativo 2010-2015, para desarrollar el programa de uso racional y eficiente de la energía y demás formas de energía no convencionales, PROURE, se definen sus objetivos y subprogramas.

La diversificación de las fuentes de energía, es otra de las formas de acabar, o mejor dicho mitigar el problema energético ambiental, por cuanto produce menos impacto negativo al ambiente $y$, además, es una salida al problema de la seguridad del suministro energético. Es importante resaltar, que la energía limpia también conocida como energía renovable, es aquella que, aprovechando los caudales naturales de energía del planeta, constituyen una fuente inagotable de flujo energético, renovándose constantemente.

De acuerdo con esto, debemos decir que el régimen jurídico y la regulación es de suma importancia para solidificar y proteger la relación entre medio ambiente, desarrollo y energía; Teniendo en cuenta lo anterior, la regulación jurídica, la planeación energéticaambiental y la política energética-ambiental, se convierten en el mejor instrumento para efectos de que la energía, el medio ambiente y el desarrollo trabajen de manera articulada y coherente.

Frente al problema energético se justifica hacer alusión a los conceptos de Ahorro Energético, Uso racional y Eficiente de la Energía, los cuales se hara alusión inmediatamente.

\section{AHORRO ENERGÉTICO, USO RACIONAL Y EFICIENTE DE LA ENERGÍA: CONCEPTO Y RÉGIMEN JURÍDICO}

El ahorro energético lo entendemos como la energía no consumida, por eso las formas que se incorporan a dicho ahorro hacen referencia a la utilización diversificada de energías limpias, diseños bioclimáticos, reutilización de recursos energéticos, utilización de aparatos de bajo consumo, reducción del consumo mediante buenas prácticas domésticas, utilización de combustibles adecuados.

En efecto, la problemática del ahorro energético se ha ido incrementando con el crecimiento de los asentamientos y los desafíos de la sustentabilidad del desarrollo territorial. La eficiencia energética es hoy un tema fundamental a nivel mundial, ya que representa un costo muy elevado en relación a los ingresos económicos que perciben los núcleos familiares por hogar (Buskañan Walker, 2008, p 12).

En lo relacionado al concepto de eficiencia energética, éste tiene como objetivo ampliar el concepto de uso racional para caracterizar la forma en que se usa la energía en el conjunto de la economía. Las mejoras de eficiencia se refieren a la reducción en cantidad de energía usada para un mismo nivel de actividad, siendo esta reducción posible por 
cambio tecnológico o cambios en la gestión y organización de un sector (Prias Caicedo, 2009, p 18).

De acuerdo con lo hasta aquí señalado, se debe decir que la Ley 1715 de 2014 define la eficiencia energetica como la relación entre la energía aprovechada y la total utilizada en cualquier proceso de la cadena energética, que busca ser maximizada a través de buenas prácticas de reconversión tecnológica o sustitución de combustibles. A través de la eficiencia energética, se busca obtener el mayor provecho de la energía, bien sea a partir del uso de una forma primaria de energía o durante cualquier actividad de producción, transformación, transporte, distribución y consumo de las diferentes formas de energía, dentro del marco del desarrollo sostenible y respetando la normatividad vigente sobre el ambiente y los recursos naturales renovables.

Es importante anotar que el ahorro de energía, eficiencia energética y uso racional de la energía son conceptos diferentes; sin embargo, todo va concatenado en aras de la protección del medio ambiente?

\subsection{FUNDAMENTO NORMATIVO DEL AHORRO ENERGÉTICO, USO RACIONAL Y EFICIENTE DE LA ENERGÍA}

Frente al tema que nos ocupa en el presente acápite del presente trabajo, es decir, el ahorro energético, la eficiencia energética y el uso racional de la energía es importante hacer alusión a la normatividad que regula la materia. Tal como lo hemos resaltado a lo largo del presente trabajo, se hizo referencia a la ley 143 de 1994, a la ley 697 de 2001 y a la ley 1715 de 2014 como soporte Legal.

El decreto 2331 de 2007 modificado y adicionado por el decreto 895 de 2008, destaca como forma de ahorro energético el cambio de bombillas incandescentes por bombillas ahorradoras, específicamente el cambio de luminarias por LFC (Lámparas Fluorescentes Compactadores) garantizando una eficiencia apreciable con adecuados niveles de iluminación y menos consumo de energía eléctrica. Este decreto tiene por objeto la utilización o sustitución, en los edificios cuyos usuarios sean entidades oficiales de cualquier orden, de todas las bombillas incandescentes por bombillas ahorradoras.

El decreto 895 de 2008 en el artículo 1 señala que en todo caso las entidades públicas de cualquier orden deberán sustituir las fuentes de iluminación de baja eficiencia lumínica por fuentes lumínicas de la más alta eficacia disponible en el mercado.

7. El concepto de eficiencia energética tiene un carácter social, técnico y económico relacionado con las acciones para reducir el consumo energético, optimizar el uso de la energía, aumentar la productividad, la competitividad y fomentar la innovación, en directa relación con aspectos tecnológicos y ambientales en el contexto del desarrollo sustentable.

8. Ley 143 de 1994. Artículo 66. (...) El ahorro de la energía, así como su conservación y uso eficiente, es uno de los objetivos prioritarios en el desarrollo de las actividades de sector eléctrico. 
En efecto, el Ministerio de Minas y Energía expidió la resolución No 180606 de 2008, la cual especifica los requisitos técnicos que deben tener las fuentes lumínicas de alta eficiencia usadas en sedes de entidades públicas. Es importante señalar que con la bombilla incandescente no se ahorra energía.

En suma, las bombillas fluorescentes compactas nos permite ahorrar el $14 \%$ con respecto a la incandescentes y la bombilla fluorescentes tubular nos permite ahorra un $83 \%$. Frente a lo dicho anteriormente, enfatizamos que el factor más relevante en el ahorro de energía y en el ahorro doméstico es la eficacia luminosa. Entre más eficaz sea una bombilla, mas ahorraremos (Guía Alumbrado Interior para edificaciones residenciales, 2007, p 3).

De igual forma, encontramos la siguiente normatividad técnica: 1. Reglamento Técnico de instalaciones eléctricas - RETIE, Ministerio de Minas y Energía de Colombia. 2 Norma Técnica Colombiana NTC 186, Icontec, 2006 sobre bombillas eléctricas de filamento de tungsteno para uso doméstico y usos similares de iluminación en general. 3. Norma Técnica Colombiana NTC 1501, Icontec, 2002 sobre Eficiencia Energética. Bombillas Fluorescentes Compactas. Rangos de desempeño energético y etiquetado. 4. Norma Técnica Colombiana NTC 1502, Icontec, 2002 sobre Eficiencia Energética. Bombillas de dos casquillos. Rangos de desempeño energético y etiquetado. 5. Norma Técnica Colombiana NTC 900, Reglas generales y especificaciones para el alumbrado público 2006-10-25.

Así mismo, la Unidad de Planeación Minero Energética expidió las guías didácticas para el buen uso de la energía, en la cual encontramos la guía del alumbrado interior de edificaciones para entidades públicas, la guía de alumbrado interior de edificaciones residenciales y la guía de alumbrado público exterior.

Al igual que en el caso Colombiano, en México existe un programa de ahorro de energía en la administración pública, cuyo propósito es reducir el gasto de energía eléctrica en las edificaciones del gobierno e inducir a conocimientos técnicos y metodologías de URE como efecto demostrativo en los funcionarios y usuarios de la administración pública federal (Prias Caicedo, 2009, p. 23).

En Colombia la eficiencia energética ha sido trabajada a partir de los siguientes enfoques normativos, así: 1. En relación directa con el PROURE. 2. Para las zonas interconectadas. 3. Fuentes no convencionales de energía con relación al cambio climático. 4. En relación con los biocombustibles. 5. Para los alcoholes carburantes. 6. Para el Gas. 7 Para la Cogeneración.

En cuanto al PROURE, se expidió la ley 697 de 2001, y el decreto reglamentario 3683 de 2003, modificado por el decreto 2688 de 2008, tal como se ha reseñado a lo largo del presente trabajo, en el cual se establecen objetivos, actividades, lineamientos, responsables 
y programas específicos. De igual forma, se expidió la resolución 18609 de 2006, que define los programas que hacen parte del uso racional y eficiente de la Energía. Así mismo, encontramos el decreto 3450 de 2008, el cual señala que en el territorio de la República de Colombia, todos los usuarios del servicio de energía eléctrica sustituirán, conforme a lo dispuesto en el presente decreto, las fuentes de iluminación de baja eficacia lumínica, utilizando las fuentes de iluminación de mayor eficacia lumínica disponibles en el mercado. De igual manera, el decreto en mención señala una prohibición expresa, en cuanto a que no se permitirá en el territorio de la República de Colombia la importación, distribución, comercialización y utilización de fuentes de iluminación de baja eficacia lumínica.

En este mismo sentido, la ley 1715 de 2014 en su capitulo V sobre desarrollo y promoción de la gestión eficiente de la energía expresa que en desarrollo del Programa de uso racional y eficiente de la energía y demás formas de energía no convencionales, PROURE, el Ministerio de Minas y Energía, conjuntamente con el Ministerio de Ambiente y Desarrollo Sostenible y el Ministerio de Hacienda y Crédito Público deberán desarrollar instrumentos técnicos, jurídicos, económico-financieros, de planificación y de información, entre los que deberán contemplarse: a) Plan de acción indicativo para el desarrollo del PROURE; b) Reglamentaciones técnicas; c) Sistemas de etiquetado e información al consumidor sobre la eficiencia energética de los procesos, instalaciones y productos y sobre el consumo energético de los productos manufacturados; d) Campañas de información y concientización.

Según la Ley 1715 de 2014 en el artículo 27, el plan de acción indicativo para el desarrollo del PROURE es el instrumento que el Gobierno utilizará para promocionar la eficiencia energética, el cual servirá para la concreción de las actuaciones en esta materia, el establecimiento de plazos para la ejecución de las mismas, la atribución de responsabilidades en el ámbito de las Entidades Públicas y la identificación de las diferentes formas de financiación y necesidades presupuestarias. De igual forma, el Estado deberá adoptar planes de gestión eficiente de la energía, que incluirán acciones en eficiencia energética y mecanismos de respuesta de la demanda.

En cuanto a las Zonas Interconectadas, encontramos la ley 633 de 2000, reglamentada por el decreto 1124 de 2008, modificado por el Decreto 4813 de 2008. Asimismo, encontramos la ley 1099 de 2006. Dicha normatividad resalta que los recursos del Fondo de Apoyo Financiero para la Energización de las Zonas No Interconectadas -FAZNI-, y los rendimientos que generen la inversión temporal de sus recursos, se utilizarán de acuerdo con la ley y con las políticas de energización que para las zonas no Interconectadas, para financiar planes, programas y/o proyectos priorizados de inversión para la construcción e instalación de la nueva infraestructura eléctrica y para la reposición o la rehabilitación de la existente, con el propósito de ampliar la cobertura y procurar la satisfacción de la demanda de energía en las Zonas No Interconectadas. De igual forma, la ley 812 de 2003 señaló expresamente, que: 
Los subsidios destinados a las Zonas no Interconectadas (ZNI) podrán ser utilizados tanto para inversión como para cubrir los costos del combustible requerido por las plantas de generación eléctrica en estas zonas. El Gobierno Nacional establecerá una metodología de asignación de dichos recursos que concertará las iniciativas de la empresa beneficiada. Parágrafo. Una vez un municipio sea interconectado al sistema Eléctrico Nacional o Internacional, el cincuenta por ciento (50\%) del subsidio que le correspondía pasará de manera automática a la municipalidad no interconectada del mismo departamento que le siga en población en su cabecera municipal, y que a su vez preste el servicio de energía eléctrica con plantas o electrógenos alimentados por combustible.

En cuanto a las fuentes no convencionales de energía en relación con el cambio climático, encontramos la Resolución 181401del 29 de octubre de 2004 modificada por la Resolución181462 de 2004, en la cual se adopta el factor de emisión de 0.471 kg CO $/$ kWh para el cálculo de las reducciones de emisiones de gases de efecto invernadero para los proyectos de generación de energía con fuentes no convencionales de energía o renovables, tales como fotovoltaica, hidroeléctrica, mareomotriz, eólica, geotérmica y biomasa, interconectados a la red, cuya capacidad instalada sea igual o menor a $15 \mathrm{MW}$, de acuerdo con la metodología del Mecanismo de Desarrollo Limpio.

En cuanto a los Biocombustibles, en los cuales ahondaremos un poco más en el presente trabajo, encontramos la Ley 693 de 2001, la cual incorpora normas sobre el uso, producción, y consumo de alcohol carburante para reducir las emisiones de gases de efecto invernadero producido por los motores de combustión.

La norma en comento señala en el artículo 1, que las gasolinas que se utilicen en el país en los centros urbanos de más de 500.000 habitantes tendrán que contener componentes oxigenados tales como alcoholes carburantes, en la cantidad y calidad que establezca el Ministerio de Minas y Energía, de acuerdo con la reglamentación sobre control de emisiones derivadas del uso de estos combustibles y los requerimientos de saneamiento ambiental que establezca el Ministerio del Medio Ambiente para cada región del país. En los centros urbanos de menos de 500.000 habitantes, el Gobierno podrá implementar el uso de estas sustancias. Ello, sin perjuicio de las demás obligaciones que sobre el particular deban observarse por parte de quienes produzcan, importen, almacenen, transporten, comercialicen, distribuyan o consuman gasolinas motor y/o combustible diésel en el país. Si el oxigenado a utilizar es Etanol carburante éste podrá ser utilizado como combustible. De igual forma, encontramos la ley 939 de 2004, que estimula la producción y la comercialización de biocombustible de origen animal y vegetal para uso en motores diésel.

En cuanto los alcoholes carburantes, encontramos la ley 788 de 2002, la cual introduce modificaciones al estatuto tributario consistentes en incentivos tributarios para los alcoholes carburantes, incentivos para proyectos de reducción de gases de efecto invernadero, donde se establece que la venta de energía no convencional por empresas 
generadoras estarán exentas de rentas por 15 años, siempre que el proyecto genere y venda certificados de reducción de GEI y destine a obras de beneficios sociales el $50 \%$ de los recursos obtenidos por este concepto.

En este mismo sentido, se introduce la Ley 863 de 2003, establece exención de impuesto al alcohol carburante, con destino a la mezcla de la gasolina para los vehículos.

En cuanto al gas, encontramos la ley 1151 de 2007, la cual en el artículo 62 señala expresamente como servicio público domiciliario al Gas Licuado ${ }^{9}$. Así mismo, se hace referencia a la cuota de fomento de gas Natural a que se refiere el artículo 15 de la ley 401 de 1997, modificado por el artículo $1^{\circ}$ de la ley 887 de 2004, la cual será del 3\% sobre el valor de la tarifa que se cobre por el gas objeto del transporte, efectivamente realizado.

Por último, en cuanto a la cogeneración, se expidió la ley 142 de 1994 modificada por la ley 1215 de 2008. La ley 1215 de 2008, resalta que quienes produzcan energía eléctrica como resultado de un proceso de cogeneración, entendido este como la producción combinada de energía eléctrica y energía térmica, que hace parte integrante de su actividad productiva, podrán vender excedentes de electricidad a empresas comercializadoras de energía, esta venta quedará sujeta a la contribución del $20 \%$ en los términos establecidos en la ley 142 de 1994, artículo 89 numeral 1 y 2. El cogenerador estará exento del pago del factor pertinente del $20 \%$ que trata este artículo, sobre su propio consumo de energía proveniente de su proceso de cogeneración.

\subsection{EFICIENCIA ENERGÉTICA Y USO RACIONAL DE LA ENERGÍA EN MATERIA DE ALUMBRADO PÚBLICO}

En el presente trabajo, es de suma trascendencia hacer alusión así sea sumariamente a la eficiencia energética en materia de alumbrado público, no sin antes referirnos a su régimen legal. La resolución 043 de 1995, de la Comisión de Regulación de Energía y gas en el artículo 1 señala que el alumbrado público es el servicio público consistente en la iluminación de las vías públicas, parques públicos, y demás espacios de libre circulación que no se encuentren a cargo de ninguna persona natural o jurídica de derecho privado o público, diferente del municipio, con el objeto de proporcionar la visibilidad adecuada para el normal desarrollo de las actividades tanto vehiculares como peatonales. También se incluirán los sistemas de semaforización y relojes electrónicos instalados por el

9. Dentro del término de dieciocho (18) meses siguientes a la expedición de esta ley, la Comisión de Regulación de Energía y Gas, CREG, adoptará los cambios necesarios en la regulación para que la remuneración asociada a la reposición y el mantenimiento de los cilindros de gas licuados de petróleo y de los tanques estacionarios utilizados para el servicio público domiciliario sea incorporado en la tarifa, introduciendo además un esquema de responsabilidad de marca en cilindros de propiedad de los distribuidores que haga posible identificar el prestador del servicio público de Gas Licuado de Petróleo que deberá responder por la calidad y seguridad del combustible distribuido. El margen de seguridad de que trata el artículo 23 de la Ley 689 de 2001 , se eliminará a partir del 31 de diciembre de 2010. A partir de la entrada en vigencia de la regulación prevista en el inciso anterior, el margen de seguridad de que trata el artículo 23 de la Ley 689 de 2001, se destinará a la financiación de las actividades necesarias para la implementación del cambio de esquema, con sujeción a la reglamentación que para el efecto expida la CREG. Y su monto se integrará al margen de distribución del servicio domiciliario del gas licuado de petróleo. 
Municipio. Por vías públicas se entienden los senderos peatonales y públicos, calles y avenidas de tránsito vehicular.

El Decreto 2424 de 2006, sostiene, en cuanto al concepto normativo de alumbrado púbico que:

es el servicio público no domiciliario que se presta con el objeto de proporcionar exclusivamente la iluminación de los bienes de uso público y demás espacios de libre circulación con tránsito vehicular o peatonal, dentro del perímetro urbano y rural de un municipio o Distrito. El servicio de alumbrado público comprende las actividades de suministro de energía al sistema de alumbrado público, la administración, la operación, el mantenimiento, la modernización, la reposición y la expansión del sistema de alumbrado público.

El alumbrado público es una herramienta valiosa para colocar en práctica el uso racional de la energía, y se hace utilizando luminarias de potencias adecuadas y en cantidades suficientes para lograr niveles de iluminación óptimos. También se regula y se hace URE cuando se mantienen las instalaciones del alumbrado público en buen estado, velando por su seguridad y sobre todo cuando se realizan actividades de limpieza a las luminarias para evitar que la capa de suciedad haga eficiente el uso de la energía. Una solución para reducir el consumo de energía por alumbrado público es el cambio de tecnología, pasando de bombillas de mercurio a bombillas de sodio de alta presión, los cuales son de mayor eficiencia y menor potencia (Upme. Guía Alumbrado Público Exterior, 2007, p 12).

\subsection{LINEAMIENTOS Y ESTRATEGIAS RELEVANTES DEL ESTADO COLOMBIANO EN MATERIA DE URE}

En el presente punto analizaremos los documentos más relevantes y de gran trascendencia en la política pública Colombiana en materia de URE, para lo cual nos limitaremos a señalar en primer lugar, el Informe final, definición de lineamientos y prioridades como apoyo a la formulación del PROURE. En segundo lugar, el documento Conpes 3510, y por último, el Plan de Acción Indicativo 2010-2015, para desarrollar el programa de Uso Racional y Eficiente de la Energía y demás formas de Energías No Convencionales.

\subsubsection{Informe final definición de lineamientos y prioridades como apoyo a la formulación del PROURE (2008)}

El informe final sobre definición de lineamientos y prioridades como apoyo a la Formulación del PROURE señala que el cumplimiento de lo establecido en el marco legal no es acatado en su totalidad por las entidades como tampoco por las empresas de servicios públicos en general. El documento de la referencia señala como posible causa de lo anterior, la dispersión y abundancia de directrices que distraen del objetivo fundamental y no define 
responsabilidades claras ni criterios unificados. Los decretos y resoluciones promulgados en el periodo de 2001 y 2008 han sido orientados a la promoción de los biocombustibles con poca atención a las fuentes no convencionales de energía.

De igual forma, el informe en mención señala que las Empresas de Servicios Públicos E.S.P han realizado esfuerzos importantes, pero aún son discretos y en algunos casos con objetivos indirectamente relacionados con la eficiencia energética; es decir, se dispone de leyes, decretos y resoluciones, experiencias puntuales desarrollos de proyectos y realización de estudios, pero se carece de lineamientos y estrategias y definición de criterios para establecer prioridades estratégicas para el país.

En el informe aludido se resalta la importancia de un programa de eficiencia energética de alcance nacional. Resalta que dicho programa es una de los mecanismos de mayor impacto e importancia que permite asegurar el abastecimiento energético, la competitividad de la economía nacional, la protección del consumidor y la promoción de las fuentes energéticas no convencionales, como un asunto de interés social público y de conveniencia nacional.

\subsubsection{Documento Conpes 3510 de 2008}

El Documento Conpes 3510 de 2008, hace referencia a los lineamientos de política para promover la protección sostenible de biocombustibles en Colombia.

El documento en comento tiene como finalidad expandir los cultivos de biomasas conocidas en el país y diversificar la canasta energética dentro de un marco de producción eficiente y sostenible económica, social y ambientalmente, que permitiera competir en el mercado nacional e internacional. Se señala que el Ministerio de Minas y Energía debe adoptar las medidas necesarias para que en todo el país la gasolina contenga un $10 \%$ de alcohol carburante y en el diésel contenga un $5 \%$ de biodiesel.

Sobre el particular, el PND ${ }^{10}$ establece que el MAVDT ${ }^{11}$ promoverá la articulación de las estrategias de prevención y control de la contaminación del aire, con la promoción por parte del gobierno de los combustibles más limpios, entre ellos, los provenientes de cultivos con potencial de producción de biodiesel y alcohol carburante. Así mismo, resalta que la propuesta de lineamiento de política de energéticos, de enero de 2006, expresa que Colombia puede aprovechar su posición geográfica para mayor captura de energía y producción de biomasa con destino a la producción de biocombustibles, siempre y cuando no se afecten ecosistemas estratégicos que provean bienes y servicios ambientales para la sociedad, y se consideren los efectos sobre seguridad alimentaria.

10. Plan Nacional de Desarrollo.

11. Ministerio del Medio Ambiente, Vivienda y Desarrollo Territorial. 
En efecto, la $\mathrm{AlE}^{12}$ ha sostenido que la dinámica del mercado de los biocombustible está asociada con la evolución de la demanda mundial de energía primaria, en la cual los combustibles fósiles tienen la mayor participación. La demanda de la energía depende del comportamiento de factores como: 1 . El aumento de la población mundial; 2 . El crecimiento económico; 3. Los desarrollos tecnológicos que permiten maximizar la eficiencia de la producción y uso; 4. La implementación de medidas frente al cambio climático tales como el desarrollo de fuentes alternativas de energía. Así, la Agencia Internacional de Energía que en el año 2020 la demanda de energía será de 16,000 Mtoe ${ }^{13}$ que corresponden a una tasa de crecimiento del 1,7 anual.

\subsubsection{Plan de Acción Indicativo 2010-2015 para desarrollar el programa de Uso Racional y Eficiente de la Energía y demás formas de Energías No Convencionales}

Para efectos del presente plan indicativo, debemos traer a colación la Resolución No 180919 de 2010 por medio del cual se adopta el plan de acción indicativo 2010-2015 para desarrollar el programa de uso racional y eficiente de la energía y demás formas de energía no convencionales, PROURE, se definen sus objetivos, subprogramas y se adoptan otras disposiciones al respecto.

Dentro de la resolución en mención se resalta que para desarrollar el programa de uso racional y eficiente de la energía y demás formas de energía no convencionales, PROURE, se hace necesario adoptar un plan de acción indicativo 2010-2015, teniendo presente el Documento Visión Colombia II Centenario: 2019 del Departamento de Planeación Nacional, el cual contempla como parte de los grandes retos del sector minas y energía, la promoción del uso eficiente de la canasta energética nacional.

Dicho plan de acción contiene, igualmente, los objetivos, subprogramas y metas, que a partir de la fecha deben regir para el Programa de Uso Racional y Eficiente de la Energía y demás Formas de Energías No Convencionales, PROURE.

De igual forma, como finalidad de los subprogramas ${ }^{14}$ contenidos en el plan, la de actualizar y unificar criterios para la ejecución de programas y proyectos sobre Uso Racional y Eficiente de la Energía y demás Formas de Energía No Convencionales en

\footnotetext{
12. Agencia Internacional de Energía.

13. Millones de Toneladas equivalentes de Crudo.

14 Resolución 180919 de 2010. Artículo $4^{\circ}$. Definir los siguientes Subprogramas estratégicos de carácter transversal del Plan de Acción Indicativo 2010-2015 del Programa de Uso Racional y Eficiente de la Energía y demás Formas de Energía No Convencionales, PROURE:

1. Fortalecimiento institucional.

2. Educación y fortalecimiento de capacidades en Investigación, desarrollo tecnológico e innovación- I+D+i y gestión del conocimiento.

3. Estrategia financiera e impulso al mercado.

4. Protección al consumidor y derecho a la información.

5. Gestión y seguimiento de metas e indicadores.

6. Promoción del uso de Fuentes No Convencionales de Energía.
} 
Colombia, para facilitar que quienes realicen proyectos puedan acceder a los incentivos nacionales e internacionales existentes en la materia.

La Resolución No 180919 de 2010, claramente consagra que, el objetivo general del plan de Acción Indicativo 2010-2015, del PROURE, el de promover el uso racional y eficiente de la energía y demás formas de energías no convencionales, que contribuya a asegurar el abastecimiento energético pleno y oportuno, la competitividad de la economía colombiana, la protección al consumidor y la promoción del uso de energías no convencionales de manera sostenible con el ambiente y los recursos naturales.

\section{LOS BIOCOMBUSTIBLES COMO ESTRATEGIA DE PROMOCIÓN DEL USO RACIONAL Y EFICIENTE DE LA ENERGÍA}

En el presente punto, empezaremos por decir que las energías alternativas pueden ser renovables o no a diferencia de las energías de origen fósil. Las primeras son aquellas energías que provienen de una fuente energética primaria de carácter renovable, entendidas como tales aquellas que no se agotan con su uso o que tienen una capacidad de regeneración de tal naturaleza que concierta anticipación y a corto plazo permiten nuevamente su utilización. En tanto que las segundas, las energías alternativas no renovables, son aquellas que tiene como fuente energética primaria las que se agotan con su uso (Hernández Mendible, 2011, p 152).

En cuanto a las clases de energía renovables, debemos hacer alusión a los biocombustibles que son los que interesan para efectos del presente acápite. Los biocombustibles e hidrocarburos tienen en común que se pueden utilizar como combustibles pero se diferencian en que los primeros tiene origen biológico, se obtienen de restos orgánicos como el azúcar, trigo, el maíz o las semillas oleaginosas y pueden ser renovables; los segundos tiene origen fósil y son no renovables (Hernández Mendible, 2011, p 154).

Tal como lo señala Hernández Mendible, los biocombustibles son combustibles de origen biológicos, pueden sustituir a los combustibles de origen fósil o incluso utilizarse mezclados con ellos, y se obtienen a través de la transformación o fermentación de las materias biológicas.

La doctrina especializada ha clasificado los biocombustibles en primera y segunda generación. En cuanto a los biocombustibles de primera generación, tenemos: 1. El biodiesel. 2. El bioetanol. 3. La biomasa. 4. Biogás. En cuanto a los biocombustibles de segunda generación, Hernández Mendible ha sostenido que se ha estado trabajando en la producción de nuevos hidrocarburos "sintéticos" - en lugar del bioetanol que presenta múltiples inconvenientes - que también se obtiene de la fermentación de azucares y el empleo de microbios o bacterias, que mediante reacciones metabólicas generan ácidos grasos que por su estructura química se asemeja más a la gasolina, el gasóleo, o el querosén. 
Para efectos del tema que nos ocupa, centraremos nuestro estudio en el presente acápite a la normatividad de carácter reglamentaria referente al alcohol carburante y la reglamentación del biodiesel.

\subsection{REGLAMENTACIÓN ALCOHOL CARBURANTE}

En cuanto al tema del alcohol carburante, es importante traer a colación la Resolución No 1565 de 2004 que regula los criterios ambientales de calidad de los combustibles líquidos y sólidos utilizados en hornos y calderas de uso comercial e industrial y en motores de combustión interna, expedida por el Ministerio de Ambiente, Vivienda y Desarrollo Territorial y el Ministerio de Minas y Energía, la cual señala que la calidad del etanol anhidro combustible, etanol anhidro combustible desnaturalizado, gasolinas básicas y gasolinas oxigenadas.

Esta resolución señala que el etanol anhidro combustible desnaturalizado antes de mezclar con las gasolinas motor, las gasolinas básicas para mezclar con etanol anhidro combustible y las gasolinas oxigenadas con etanol anhidro combustible que se produzcan, importen o distribuyan por cualquier persona natural o jurídica para el consumo dentro del territorio colombiano, deberán cumplir todos y cada uno de los requisitos de calidad señalados en las respectivas Tablas $1 \mathrm{~A}, 1 \mathrm{~B}, 2 \mathrm{a}$ y $2 \mathrm{~B}$.

Sobre el particular, es oportuno, además, hacer alusión al decreto 2629 de 2007, por medio del cual se dictan disposiciones para promover el uso de biocombustibles en el país, así como medidas aplicables a los vehículos y demás artefactos a motor que utilicen combustibles para su funcionamiento. La norma fue modificada por el decreto 1135 de 2009, y establece como estrategia de promoción que los vehículos automotores hasta $2000 \mathrm{~cm} 3$ de cilindrada que se fabriquen, ensamblen, importen, distribuyan y comercialicen en el país y que requieran para su funcionamiento gasolinas, deberán estar acondicionados para que sus motores funcionen con sistema Flex-fuel (E85), es decir, que puedan funcionar normalmente utilizando indistintamente gasolinas básicas o mezclas compuestas por gasolina básica de origen fósil con al menos $85 \%$ de alcohol carburante. Lo anterior, empezó a regir a partir del $1^{\circ}$ de enero del año 2012.

Para terminar, es importante hacer alusión a la Resolución No. 90705 de 2013 por medio de cual se establece el ingreso al productor del alcohol carburante y del biocombustible para uso de motores diésel a partir del 1 de septiembre de 2013.

\subsection{REGLAMENTACIÓN BIODIESEL}

En cuanto al tema delos biocombustibles, es importante recordar la resolución No 18 2087 de 2007,por la cual se modifica parcialmente la resolución 898 del 23 de agosto de 1995, que regula los criterios ambientales de calidad de los combustibles líquidos 
y sólidos utilizados en hornos y calderas de uso comercial e industrial y en motores de combustión interna expedida por el Ministerio del Medio Ambiente, Vivienda y Desarrollo Territorial y el Ministerio de Minas y Energía, en la cual se expresa que el biocombustible que deberá ser utilizado para mezclar con los combustibles diésel fósiles y el combustible diésel regular y sus mezclas, que se produzcan, importen o distribuyan por cualquier persona natural o jurídica, para consumo en el territorio colombiano, excepto en la ciudad de Bogotá, D. C., deberá cumplir todos y cada uno de los requisitos de calidad especificados en dichas Tablas 1A, 1B, 2A, y 2B.

Sobre el tema del biodiesel, existen, además, entre otras, las resoluciones No. 180242 de 2008, por el cual se expiden normas para el registro de productores y/o importadores de biocombustibles para uso en motores diésel y se establecen otras disposiciones en relación con su mezcla con el ACPM de origen fósil, modificada por la resolución No. 180243 de 2008, que tiene por objeto establecer los requisitos técnicos y de seguridad para la producción e importación de biocombustibles para uso en motores diésel y de sus mezclas con el diésel (ACPM) de origen fósil, de conformidad con lo dispuesto en la ley 939 de 2004, así como señalar otros aspectos importantes para la distribución de las señaladas mezclas.

La resolución No 180242 de 2008, expresamente señala en el artículo 4 el porcentaje de mezcla por el tipo de mezclador, lo siguiente:

Los Refinadores y/o importadores serán los encargados de realizar la mezcla del $2 \%$ del biocombustible para uso en motores diésel con el diésel (ACPM) de origen fósil. De otro lado, el distribuidor mayorista será el encargado de realizar la mezcla del tres por ciento (3\%) adicional, con el fin de que se distribuya y en concordancia con lo señalado en el artículo anterior, mezclas de un cinco por ciento (5\%) de biocombustible para uso en motores diésel con un noventa y cinco por ciento (95\%) de diésel (ACPM) de origen fósil (B-5).

Así mismo, es importante hacer alusión a la resolución 181556 de 2010,por la cual se modifica la Resolución 182439 del 23 de diciembre de 1998, y se establecen disposiciones relacionadas con la estructura de precios del ACPM y de la mezcla del mismo con el biocombustible para uso en motores diésel.

De igual forma, encontramos la resolución No 182142 de 2007, modificada por la resolución No 181120 de 2010, y modificada por la Resolución No. 91662 de 2012, la cual regula el programa de mezcla de biocombustible para uso en motores diésel.

Para terminar, es importante mencionar el decreto 4892 de 2011 por el cual se dictan disposiciones aplicables al uso de alcoholes carburantes y biocombustibles para vehículos automotores. 
De la norma en comentario, es oportuno hacer alusión textualmente a lo siguiente:

Artículo $1^{\circ}$. A partir de la entrada en vigencia de este Decreto se utilizarán en Colombia los siguientes combustibles, en lo que a motores a gasolina se refiere:

1. Gasolina motor con porcentajes de mezcla obligatoria que variarán entre el $8 \%$ y el $10 \%$ de mezcla de alcohol carburante en base volumétrica (E-8 -E-10 corriente y extra). A partir del $1^{\circ}$ de enero del año 2013, los ministerios de Minas y Energía y de Ambiente y Desarrollo Sostenible, o quien haga sus veces y mediante acto administrativo, previa consulta con la Comisión Intersectorial de Biocombustibles, podrán fijar porcentajes obligatorios de alcohol carburante superiores al 10\% de mezcla obligatoria para el alcohol carburante.

2. Para uso en motores diésel, a partir del $1^{\circ}$ de enero del año 2013 , los ministerios de Minas y Energía y de Ambiente y Desarrollo Sostenible, o quien haga sus veces y mediante acto administrativo, previa consulta con la Comisión Intersectorial de Biocombustibles, podrán fijar porcentajes obligatorios de biocombustibles superiores al $10 \%$ de mezcla obligatoria de biocombustibles. Parágrafo. Los ministerios de Minas y Energía y de Ambiente y Desarrollo Sostenible, o quien haga sus veces, tomarán en cuenta (i) la oferta nacional de alcohol carburante y de biocombustibles para uso en motores diésel; (ii) en la medida en que tecnológica y ambientalmente sea viable para el parque automotor, y, (iii) se tenga claridad sobre la infraestructura asociada al almacenamiento, transporte y distribución.

3. En forma voluntaria, y sin perjuicio de lo señalado sobre mezclas obligatorias en los incisos anteriores, para vehículos con tecnología Flex Fuel exclusivamente (E-25 E 85), gasolina motor con una mezcla flexible de alcohol carburante entre un $25 \%$ y un $85 \%$ en base volumétrica.

En síntesis, la estructura del sistema energético colombiano prioriza y se sostiene en los combustibles fósiles, haciendo de ellos su eje central, de otro lado, el Estado Colombiano no ha propuesto mecanismos regulativos sólidos y estructurales para hacer de las energías renovables a mediano y largo plazo las energías del futuro de su mercado energético de manera competitiva. Es claro que las estrategias de fomento son necesarias pero no suficientes frente al problema del suministro energético, la sostenibilidad del sistema energético y la protección ambiental. Es claro que se debe incorporar dentro de la canasta energética, energías limpias que compitan frente a los combustibles fósiles, a partir de la facultad regulativa estatal como prerrogativa pública. El sistema jurídico energético colombiano no incluye en la canasta energética a las energías renovables como componente central, por cuanto la normatividad nacional solo se limita a fomentar las energías renovables sin implementar reformas estructurales al sistema en aras de hacer de las energías renovables el elemento central de la economía colombiana.

\section{CONCLUSIONES}

Frente al problema jurídico planteado, esto es, cual ha sido el tratamiento jurídico de las energías renovables en Colombia y cual ha sido el nivel de incidencia de estas, en 
la protección ambiental, es necesario señalar que sí bien ha desarrollado tímidamente el tema de los biocombustibles como energías renovables, la propuesta frente a otras fuentes no convencionales ha sido deficiente, porque no ha estructurado herramientas eficaces para entregar competitividad dentro del mercado energético a las energías renovables, siendo pasivo el Estado pasivo frente a la protección ambiental en cuanto a este punto se refiere.

La regulación energética es una herramienta jurídica eficiente para hacer de las energías renovables un recurso principal en el sistema energético tanto global como colombiano, para efecto de procurar la competitividad de dichas energías en el mercado energético.

El sistema jurídico energético colombiano no incluye en la canasta energética a las energías renovables como componente central, por cuanto la normatividad nacional solo se limita a fomentar las energías renovables sin implementar reformas estructurales al sistema en aras de hacer de las energías renovables el elemento central de la economía colombiana. En otras palabras, la política energética ambiental y del sistema jurídico energético, en materia de energías renovables es deficiente debido a la falta de centralización de las energías renovables como eje del sistema energético, impide la competitividad de dichas energías en el mercado energético.

Es claro que se debe incorporar dentro de la canasta energética, energías limpias que compitan frente a los combustibles fósiles, a partir de la facultad regulativa estatal como prerrogativa pública. El sistema jurídico energético colombiano no incluye en la canasta energética a las energías renovables como componente central, por cuanto la normatividad nacional solo se limita a fomentar las energías renovables sin implementar reformas estructurales al sistema en aras de hacer de las energías renovables el elemento central de la economía colombiana.

El papel de las autoridades energéticas y ambientales frente a los problemas del sector energético es fundamental y debe orientarse primeramente hacia la fijación de políticas que definan el rumbo del sector energético, a partir de la edificación de una estructura energetica que permita el ingreso dentro de la canasta energetica a las energias renovables de manera competitiva.

\section{REFERENCIAS BIBLIOGRÁFICAS}

Alba Hidalgo, D., Menendez Perez, E., \& Ramirez Piris, N. (n.d.). Energia, Medioambiente y Desarrollo Sostenible en la Unión Europea. 1-12.

Alvarez Gomez, J., \& Alonso Gonzalez, A. Noción de Crecimiento y Desarrollo Economico. Revista Galega de Economia, 2006, 1-10. 
Buskañan Walker, F. Efectos de la entropia urbana en el coste energetico del transporte urbano, 20-27.

Beato, P., \& Merino, P. A. Las necesidades de intervención en el sector energetico en un contexto de crecimiento economico. In C. N. Energia, Energia y Regulación en Iberoamerica, Pamplona: Thomson-Civita, 2008, p. 869.

Betancourt, L. I. Perspectiva. Energias Renovables: Marco Juridico en Colombia, 2009, p. 69-71.

Caballero Miguel, G. Economia Ambiental: Perspectiva Institucional. Revista Galena Economica, Vol, 11 No. (2), 2002, 1-13.

Carrillo Rodriguez, J. (2009). Politicas Públicas Regulativas. Teoria y experiencia en el pilar ambiental. In Regulación Energetica y Medio Ambiente, 2009 p. 133-147. Barranquilla: Ediciones Uninorte.

Ceballo Ángel, L. I., \& Valbuena Pájaro, M. A Los biocombustibles y los problemas asociados al medio ambiente y la seguridad alimentaria. Fragua, 2010, p. 35-43.

Centro Complutense de Estudios e Información Medioambiental, Cambio Global España 2020/50. Energía, economía y sociedad. Asturias, 2011.

Centro Superior de estudios de la Defensa Nacional. La Crisis Energética y su repercusión en la Economía. Seguridad y Defensa Nacional, 2008.

Comisión Brundland. Nuestro Futuro Común, 1987.

Corominas, J. Crisis Energética y Cambio Climático: iProblema u Oportunidad? In e. a. Capdevila Laia, Cambio Climático y Crisis Energetica, Catalunya, Graficos El Rey S.L., 2008, pp. 55-66,

Del Rio Gonzalez, P. Los Efectos sobre la Innovación de lo Instrumentos de Promoción de Ias Energias Renovables. Cambio Climatico: Aspectos Economicos e Internacionales, 2011, 43-56.

Durán Ruiz, F. J. (2009). La imbricación entre la politica energetica y ambiental en la Unión Europea y las energias renovables. In Regulación Energetica y Medio Ambiente (pp. 88-114). Barranquilla: Ediciones Uninorte.

Estrada Gasca, C., \& otro, y. Las Energias renovables: La Energia Solar y sus implicaciones. Revista Digital Universitaria, 2010, 1-27. 
Fernandez Sandoval, P. Reto Europeo: La eficiencia energetica en edificios, 2011, p 1-23.

Gonzalez Arias, A. El concepto de energia en la enseñanza de las ciencias, Revista Iberoamericana de Educuación, p 1-6.

Hernadez Martin, M. A. El sector Energetico en Europa: Hacia una Europa mas verde. In U. d. Norte, Regulación Energetica y Medio Ambiente 2009, pp. 265-293. Barranquilla: Uninorte.

Hernández Mendible, Víctor, "Regulación comparada de energías renovables" Bogotá, Universidad Externado de Colombia, 2013, p 257.

Iranzo Martín, J. E., \& Colinas González, M. La Energía en España: Un reto estrategico. Vol., 282, 2008, 141-154.

Jimenez Beltran, D., "Energy and environment in the European Union" Luxembourg, en Office for Official Publications of the European Communities, No. 31, 2002.

Martinez Corcoles, F. Las Energias Nuclear y Renovables en la Cesta del Suministro Energetico, 2010, 95-99.

Martinez Fernandez, M. Planeación Energetica en Mexico y sus futuros. Revista Digital Universitaria, 2010, 1-24.

Marin Hernandez, Hugo A. "Norma Regulatoria": ¿mito o realidad? Revista Digital de Derecho Administrativo Vol. 3, 2009, 27-66.

Mera Vasquez, M. T. Teoria Economica de los Derechos de Propiedad. In M. T.

Mera Vasquez, \& F. Gomez Camacho, De economía y pensamiento economico, España, 2008, pp. 389-408.

Ramirez Laureano, E., \& otro, y. El uso de energia limpia y su importancia en la conservación del medio ambiente, Ediciones Madrid, Nueva Juridica, 2008.

Sanchez Perez, German, Desarrollo y medio ambiente. una mirada a Colombia. Economia y Desarrollo, Vol 1, 2002, pp. 79-98.

Velasquez Muñoz, Carlos. J. Analisis de los desarrollos politico-normativos realizados por la Union Europea y el Estado Colombiano para el fomento y uso de las energias alternativas. In Regulacion Energetica y Medio Ambiente, Barranquilla, Ediciones Uninorte, 2009, pp. 27-71. 
Velasquez Muñoz, Carlos. El desarrollo Humano sostenible como mandato constitucional: fundamentos axiologico-normativos vinculante o simple retorica. Barranjquilla, Ediciones Uninorte, 2010.

Villanueva Monzon, M., \& Hialgo Nuchera, A. El despliegue de las Energias Renovables en España. Necesidad de acciones de politica técnologica. Economia Industrial, 2003, 103-113. 